\title{
Critical Casimir Forces and Colloidal Phase Transitions in a Near-Critical Solvent: A Simple Model Reveals a Rich Phase Diagram
}

\author{
John R. Edison, ${ }^{1}$ Nikos Tasios, ${ }^{1}$ Simone Belli, ${ }^{2}$ Robert Evans, ${ }^{3}$ René van Roij, ${ }^{2}$ and Marjolein Dijkstra ${ }^{1,}$ \\ ${ }^{1}$ Soft Condensed Matter, Utrecht University, Princetonplein 5, 3584 CC Utrecht, Netherlands \\ ${ }^{2}$ Institute for Theoretical Physics, Utrecht University, Leuvenlaan 4, 3584 CE Utrecht, Netherlands \\ ${ }^{3}$ H. H. Wills Physics Laboratory, University of Bristol, Bristol BS8 1TL, United Kingdom \\ (Received 12 September 2014; revised manuscript received 4 November 2014; published 21 January 2015)
}

\begin{abstract}
From experimental studies, it is well known that colloidal particles suspended in a near-critical binary solvent exhibit interesting aggregation phenomena, often associated with colloidal phase transitions and assumed to be driven by long-ranged solvent-mediated (SM) interactions (critical Casimir forces), set by the (diverging) correlation length of the solvent. We present the first simulation and theoretical study of an explicit model of a ternary mixture that mimics this situation. Both the effective SM pair interactions and the full ternary phase diagram are determined for Brownian disks suspended in an explicit two-dimensional supercritical binary liquid mixture. Gas-liquid and fluid-solid transitions are observed in a region that extends well away from criticality of the solvent reservoir. We discuss to what extent an effective pairpotential description can account for the phase behavior we observe. Our study provides a fresh perspective on how proximity to the critical point of the solvent reservoir might influence colloidal self-assembly.
\end{abstract}

DOI: 10.1103/PhysRevLett.114.038301

Colloidal particles dispersed in a binary solvent mixture have an inherent preference for one of the two solvent species. This is reflected by preferential adsorption of the favored species on the colloid surface, leading to the development of adsorbed films. Such films can mediate an effective interaction between two colloidal particles which is remarkably sensitive to the thermodynamic state of the solvent. Close to the (demixing) critical point of the solvent, the adsorbed film thickness is determined by the correlation length $\xi$ of the solvent [1], and, as first predicted by Fisher and de Gennes [2], the resulting solvent-mediated (SM) interactions are long ranged, with universal scaling properties. An analogy between the confinement of quantum fluctuations of the electromagnetic field [3] and that of thermal composition fluctuations in a near-critical binary solvent led to these (universal) SM forces being referred to as critical Casimir forces [4].

Theoretical studies on near-critical fluids confined between a pair of infinitely large planar walls (representing two static large colloids) [4-8], along with direct experimental measurements of the Casimir force $[9,10]$ between a colloid and a wall, have advanced our understanding of the nature of two-body SM interactions. Although experimental investigations of a suspension of colloids go back to the pioneering work of Beysens and Estève [11] (for a very recent experimental study, see [12]), the theory and computer simulation of such systems remain at a primitive stage. Here we use computer simulations of a simple model to understand the strength and range of the SM interactions and the resulting phase behavior of a dense colloidal suspension as a function of the thermodynamic state of the solvent. Computer simulation of colloids in an explicit
PACS numbers: 82.70.Dd, 47.57.J-, 64.70.pv, 64.75.Xc

molecular solvent with a bulk correlation length that diverges upon approaching the critical point is notoriously difficult, as very different length and time scales are involved. Nevertheless, by sacrificing one spatial dimension and using a lattice model, we have calculated the phase diagrams for an explicit ternary solvent-solvent-colloid mixture, without resorting to the assumption of pairwise effective potentials employed in other studies, most notably Refs. [13-15].

Following Rabani et al. [16], we model the ternary solvent-solvent-colloid mixture as an incompressible $A B C$ mixture on a $2 \mathrm{D}$ square lattice, as shown schematically in Fig. 1. Colloids $C$ are discretized hard disks with a radius of $R$ lattice sites, occupying a fraction $\eta$ of the lattice sites. Every site that is left unoccupied by the colloidal disks is occupied by a solvent molecule of either species $A$ or $B$, such that the fraction of sites occupied by $A$ and $B$ equals $1-\eta-x$ and $x$, respectively. We consider only nearest neighbor $A B$ repulsions and $B C$ attractions: an energy penalty $\frac{1}{2} \epsilon>0$ is assigned to every nearest neighbor $A B$ pair to drive $A B$ demixing at sufficiently low temperatures $T$ and an energy gain $-\frac{1}{2} \alpha \epsilon$ with $\alpha \geq 0$ for every $B C$ pair to mimic the colloid $C$ 's preference for species $B$. Throughout, we set the lattice spacing to unity. We investigated carefully several lattice effects present in our model, and we discuss it briefly in Sec. V of the Supplemental Material [17]. We note that lattice effects have no implications for the key results of our work.

In the limit $\eta=0$, our model reduces to a binary $A B$ mixture that is isomorphic to the 2D lattice (Ising) model. The critical temperature of this binary mixture is $T_{c}=0.567 \epsilon / k_{B}$, and its thermodynamic state is 


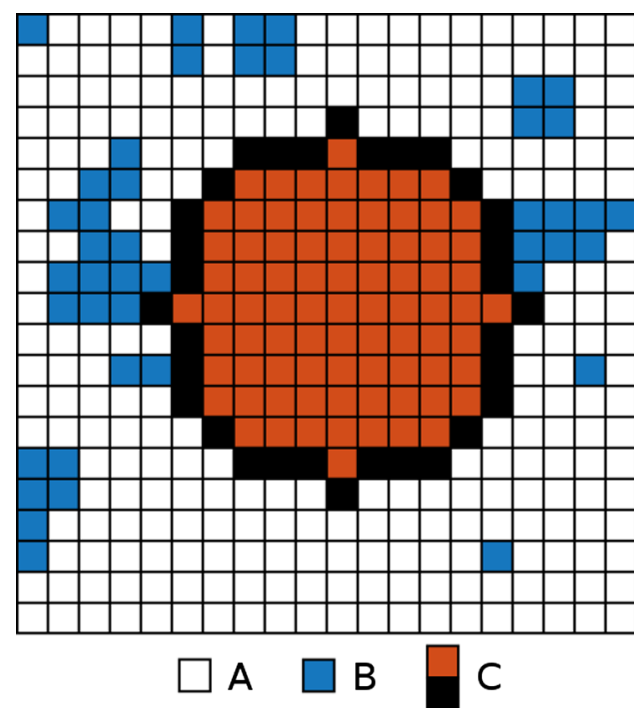

FIG. 1 (color online). A schematic representation of the solvent-solvent-colloid lattice model. White cells are occupied by solvent species $A$, blue cells by solvent species $B$, and brown and black cells represent the interior and the boundary of a single colloidal particle $C$, respectively.

characterized fully by the reduced temperature $\tau=$ $\left(T-T_{c}\right) / T_{c}$ together with either the reduced chemical potential difference $\Delta \mu_{s}=\left(\mu_{B}-\mu_{A}\right) / \epsilon$ between species $B$ and $A$ or the composition $x$. For $\Delta \mu_{s}<0$, the $A B$ mixture favors an $A$-rich composition at all temperatures. Moreover, for $\tau<0$, demixing into an $A$-rich state $(x<0.5)$ and a $B$ rich state $(x>0.5)$ takes place at $\Delta \mu_{s}=0$, with a critical point $\left\{\tau_{c}=0, x_{c}=0.5\right\}$; see Figs. 2(a) and 2(b). In the two limits $\Delta \mu_{s} \rightarrow \pm \infty$, our $A B C$ mixture reduces to the 2D $A C$ or $B C$ hard-disk system with packing fraction $\eta$ in an (irrelevant) pure $A$ solvent $(x=0)$ or pure $B$ solvent $(x=1-\eta)$. Barring small discretization and lattice artifacts, and ignoring subtleties regarding the (non)existence of a stable hexatic phase, these $A C$ and $B C$ systems exhibit fluid-solid coexistence for $\eta \in[0.700,0.716]$ as represented by vertical dashed lines in Figs. 3(a)-3(c) [24].

Throughout this work, we study colloids immersed in a supercritical (one-phase) $A B$ mixture, relatively poor in the colloid-preferred species $B(\eta>0, \tau>0$, and $\left.\Delta \mu_{s} \leq 0\right)$. This choice precludes solvent-mediated colloidal aggregation arising from complete wetting and capillary condensation [25]. The solvent is treated grand canonically; i.e., the system is in thermal and diffusive contact with an $A B$ solvent reservoir with composition $x_{r}$ that fixes $\tau$ and $\Delta \mu_{s}$. The $A B C$ mixture has composition $x \neq x_{r}$. Note that $\tau$ merely sets the temperature; it is not a measure of distance from criticality of the ternary mixture.

We focus on the case where $B$-rich layers adsorbed on the colloid surfaces compete with a supercritical $A$-rich bulk solvent ( $\tau>0$ and $\Delta \mu_{s}<0, \alpha=0.6$ ). We perform simulations of the model in the fixed $\left(\eta, \tau, \Delta \mu_{s}\right)$ ensemble (see Sec. I of the Supplemental Material [17]) and in the
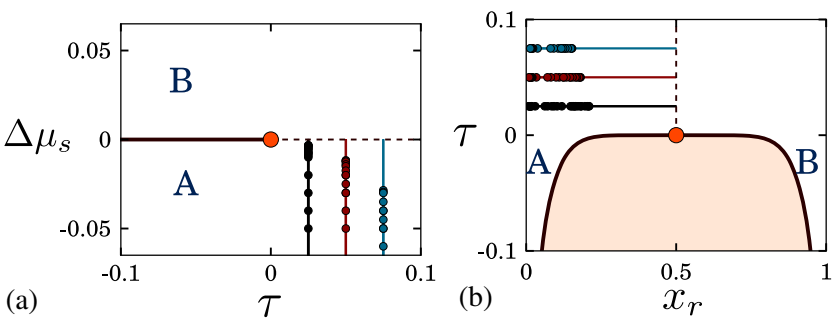

FIG. 2 (color online). (a) Phase diagram of the colloid-free $A B$ solvent mixture plotted as $\Delta \mu_{s}$ vs $\tau$. (b) Phase diagram (binodal) of the same mixture plotted as $\tau$ vs $x_{r}$, the composition of the solvent. The two coexisting phases for $\tau<0$ are designated $A$ and $B$. In (a) and (b), the lines correspond to the paths along which the phase diagram of the full ternary $A B C$ mixture is determined; the dots represent the states where phase coexistence is observed, and the orange dot indicates the critical point of the $A B$ solvent mixture $\left(\tau=0, \Delta \mu_{s}=0\right)$.

grand ensemble, using the staged insertion method [26] together with the transition matrix Monte Carlo (TMMC) technique [27], to accurately determine phase coexistence. The grand canonical (GC)-TMMC results reported here are for system size $L=256$. For a few state points, we compared the results for two different system sizes $L=$ 256 and $L=512$ and found the coexisting packing fractions to be the same up to the third decimal place. In Figs. 3(a) and 3(b), we present the phase diagram of the ternary mixture in the $\Delta \mu_{s}$ vs $\eta$ representation for $\tau=0.025,0.05,0.075$. The correlation length of the $A B$ solvent reservoir at the isochoric composition $\left(\Delta \mu_{s}=0\right.$, $\left.x_{r}=0.5\right)$ is $\xi=0.567 / \tau$, and for these temperatures it is comparable to the size of the colloid $R=6$. Although the underlying $A B$ solvent reservoir is supercritical, our simulations reveal that a nonzero concentration of large Brownian disks induces stable colloidal gas $(G)$, liquid $(L)$, and crystal $(X)$ phases as well as two-phase $G-L$ and $G-X$ coexistence. The $G-L$ coexistence, shown more clearly in Fig. 3(b), terminates at a critical point that shifts to lower $\Delta \mu_{s}$ and higher $\eta$ with increasing $\tau$. By tracing the locus of the three critical points of the $A B C$ mixture from $\tau=0.075$ through 0.05 to 0.025 , it appears that the critical points of the colloidal $G-L$ transition are continuously connected to that of the binary solvent mixture $\left(\eta=\tau=\Delta \mu_{s}=0\right)$; investigations at smaller $\tau$ are constrained by our computational resources.

For $\Delta \mu_{s}<-0.1$, we also observe $G-X$ coexistence with a broad colloid density gap that narrows sharply upon lowering $\Delta \mu_{s}$, consistent with the limiting hard-disk fluid-solid coexistence at $\Delta \mu_{s} \rightarrow-\infty$ (vertical dashed lines). Significantly, this decreasing density gap at $G-X$ coexistence suggests an additional underlying metastable $G-L$ lower critical point. Although we have not been able to identify this in our MC simulations, such an additional critical point does occur in our mean-field treatment presented in the Supplemental Material [17]. Moreover, 


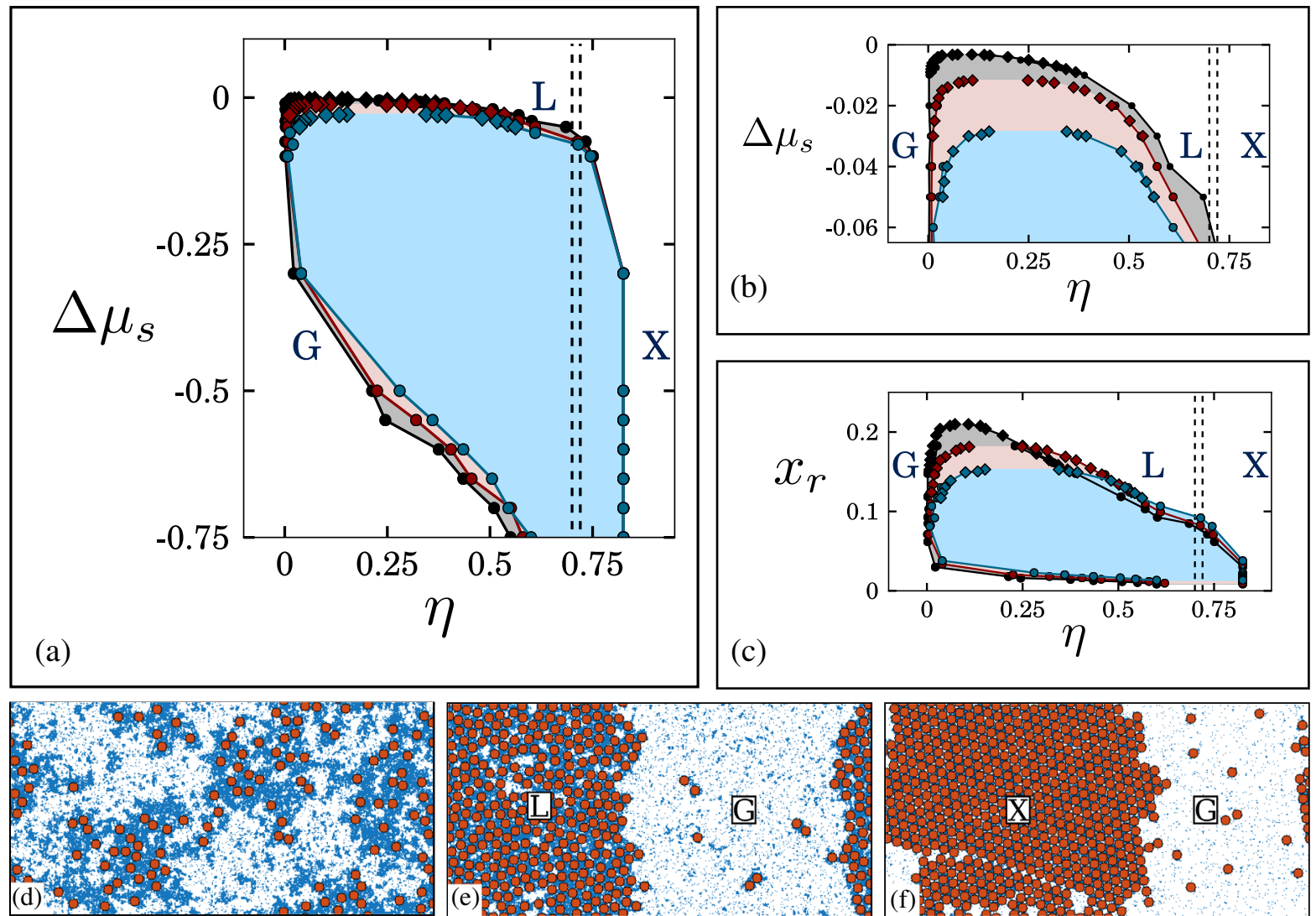

FIG. 3 (color online). (a) Phase diagrams of the full $A B C$ ternary mixture plotted as solvent chemical potential $\Delta \mu_{s}$ vs hard-disk (colloid) packing fraction $\eta$ for $R=6$. (b) The top portion of (a) is replotted for clarity. (c) Phase diagram of the $A B C$ model plotted as reservoir solvent composition $x_{r}$ vs $\eta$. Black, red, and blue symbols refer to $\tau=0.025,0.05$, and 0.075 , respectively. The diamonds and dots denote the phase boundaries obtained from grand canonical staged insertion Monte Carlo (MC) simulations and $\left(\eta, \tau, \Delta \mu_{s}\right)$ ensemble MC simulations, respectively. The vertical dashed lines denote fluid-solid coexistence for pure hard disks. (d)-(f) Simulation snapshots of a system of $256 \times 512$ lattice sites at $\tau=0.05$ and $\alpha=0.6$, showing (d) a supercritical colloidal phase at $\Delta \mu_{s}=-0.005$ of 128 colloids, (e) gas-liquid $(G-L)$ coexistence at $\Delta \mu_{s}=-0.04$ of 348 colloids, and (f) gas-crystal $(G-X)$ coexistence at $\Delta \mu_{s}=-0.3$ of 580 colloids.

if we accept hard-disk coexistence in the opposite limit $\Delta \mu_{s} \rightarrow \infty$, then we also expect a $G-L-X$ triple point at $\Delta \mu_{s} \simeq-0.06$ for $\tau=0.025$ [see Fig. 3(b)] and at even lower $\Delta \mu_{s}$ for higher $\tau$.

In Figs. 3(d)-3(f) and in the Supplemental Material's movie-03/04/05 [17], we show results for systems of $256 \times 512$ lattice sites simulated at reduced temperature $\tau=0.05(\alpha=0.6, R=6)$ that illustrate configurations of (d) a supercritical (homogeneous single-phase) fluid state, (e) $G-L$ coexistence, and (f) $G-X$ coexistence. In all three cases, the local solvent composition is strongly correlated with the local colloid density, such that the coexisting $L$ phase in (e) and $X$ phase in (f) have a binary $B C$ composition with tiny traces of $A$. Conversely, in the coexisting $G$ phases shown in Figs. 3(e) and 3(f), the solvent composition is very close to the composition of the reservoir $x \simeq x_{r}$. In Fig. 3(c), we convert the phase diagram of Fig. 3(a) into the $x_{r}-\eta$ representation. It is evident from the snapshots and Figs. 3(c) and 2(b) that, for all observed $G-L$ and $G$ - $X$ coexistence, (i) the composition of the solvent reservoir $x_{r}<0.25$ is far from its critical composition $x_{c}=0.5$, and (ii) the correlation length of the solvent is smaller than the colloid radius, $\xi<R$. Strikingly, in the homogeneous supercritical state of Fig. 3(d), the correlation length (the typical size of the $A$-rich and $B C$ rich "patches") is clearly much larger than the colloid radius and thus far exceeds that of the solvent reservoir. This reflects the nearby $(G-L)$ critical point of the ternary mixture which, as noted previously, appears to be continuously connected to the critical point of the binary $A B$ solvent mixture $\left(\eta=\tau=\Delta \mu_{s}=0\right)$. In fluid mixtures where the species interact via short-range potentials, all structural correlations decay with the same correlation length [28]. Therefore, along the $G-L$ critical locus, solvent-solvent, colloid-colloid, and solvent-colloid correlations should decay with the same, diverging correlation length. We have confirmed this numerically by calculating the $B B, B C$, and $C C$ pair correlation functions; see Fig. S3 

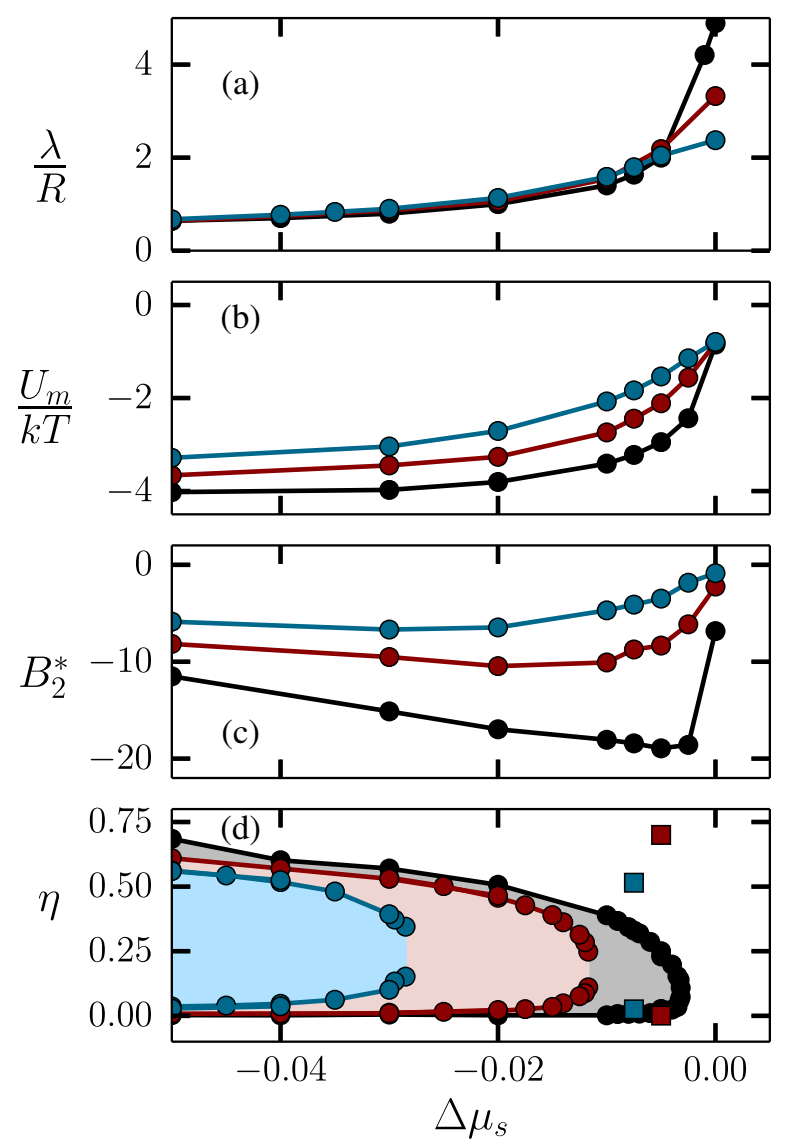

FIG. 4 (color online). (a) Thickness $\lambda$ of the $B$-rich film adsorbed on a single colloid. (b) The minimum well depth of the effective two-body SM potential. (c) The reduced second virial coefficient $B_{2}^{*}$. (d) To facilitate comparison, the phase boundaries of the ternary $A B C$ mixture of Fig. 3 are replotted in the $\eta-\Delta \mu_{s}$ representation. The square symbols (d) correspond to packing fractions of coexisting phases computed from simulations of colloids interacting via the measured two-body interaction. Black, red, and blue symbols refer to reduced temperatures $\tau=0.025$, 0.05 , and 0.075 , respectively $(R=6, \alpha=0.6)$.

of the Supplemental Material [17]. We have also confirmed the divergence of the long wavelength limit of the structure factor $S_{B B}$ upon approaching the critical point of the ternary mixture at a fixed value of temperature $\tau=0.025$; see Fig. S4 of the Supplemental Material [17].

As mentioned earlier, there have been several attempts to ascertain the phase behavior of colloids in a near-critical solvent based solely on effective two-body interactions, e.g., Refs. $[12,13]$. In order to assess the validity of this approach for the present model system, we calculated several oneand two-colloid properties for the range of thermodynamic state points studied above. For the three temperatures investigated, we show in Fig. 4 the dependence on $\Delta \mu_{s}$ of (a) the thickness $\lambda$ of the adsorbed $B$-rich film on a single disk, (b) the minimum $U_{m}$ of the effective pair potential $U(x, y)$, and (c) the reduced second virial coefficient $B_{2}^{*}=(1 / 2) \int_{-\infty}^{\infty} \int_{-\infty}^{\infty}\left\{1-\exp \left[-U(x, y) / k_{B} T\right]\right\} d x d y / 2 \pi R^{2}$, normalized to that of $2 \mathrm{D}$ hard disks. The effective two-body potential $U(x, y)$ is obtained as follows. We simulate a system of just two colloids suspended in solvent at fixed $\left\{\Delta \mu_{s}, \tau\right\}$. We fix the position of one colloid fixed at $(0,0)$ and compute the probability $P(x, y)$ of finding the other colloid at position $(x, y)$, using the TMMC technique (see Sec. V of the Supplemental Material [17] for details). To facilitate comparison, we replot the phase diagrams of Fig. 3 in the $\eta-\Delta \mu_{s}$ representation in Fig. 4(d). The film thickness $\lambda$ and the well depth $U_{\min }$ are measures of the range and strength of $U(x, y)$, respectively. The quantity $B_{2}^{*}$ is a well-established (dimensionless) measure of combined strength and range. This must be sufficiently negative in order for gas-to-liquid condensation to occur in systems described by pairwise additive interactions $[29,30]$.

Figure 4(a) shows a monotonic increase of the film thickness from $\lambda \ll R$ to $\lambda \gg R$, reflecting the growth of the correlation length, as the isochoric composition is approached $\left(\Delta \mu_{s} \rightarrow 0\right)$. In the same range, $U_{m}$ varies nonmonotonically, being strongest at slightly negative $\Delta \mu_{s}$, reaffirming earlier theoretical predictions [31,32]. At $\Delta \mu_{s} \simeq 0$, the effective pair potential is long ranged; however, it is only weakly attractive $\left(\left|U_{m}\right| / k_{B} T<1\right)$. Upon decreasing $\Delta \mu_{s}, U(x, y)$ does become more attractive, although the adsorbed film thickness $\lambda$ and thereby the range of $U(x, y)$ decrease. $B_{2}^{*}$ also becomes more negative.

We performed simulations of the effective system, with the colloid-colloid pair interaction determined by the measured effective potential. For states where the actual ternary mixture is supercritical, e.g., $\{\tau=0.075$, $\left.\Delta \mu_{s}=-0.0075\right\},\left\{\tau=0.05, \Delta \mu_{s}=-0.005\right\}$ [cf. Figs. 3(a) and 4(d)], simulations performed with the effective twobody potential predict $G-L$ coexistence. The square symbols in Fig. 4(d) denote the packing fractions of coexisting phases at these two representative state points. This along with the $B_{2}^{*}$ and $U_{m} / k T$ curves indicates that the approaches employing only effective pair potentials as obtained from, e.g., planar slit studies and the Derjaguin approximation overestimate the extent of $G-L$ coexistence and underestimate the shift in critical point of the ternary mixture with respect to that of the solvent reservoir.

In summary, we find the phase behavior of a model of colloids in a near-critical solvent to be rich; (i) we observe $G-L$ and $G-X$ coexistence with accompanying solvent demixing, (ii) both occur far from the critical point of the solvent reservoir and the locus of $G-L$ critical points appears to connect smoothly to this, and (iii) many-body interactions are crucial to account quantitatively for the observed colloidal phase behavior. In light of our results, it would be interesting to revisit the problem of protein assembly in two-dimensional plasma membranes of living cells $[33,34]$ and the recent experiments of Nguyen et al. [12]. The topology of the phase diagram of colloidal particles in a near-critical binary solvent stems from an intricate balance between competing colloid-solvent and 
solvent-solvent couplings that can be captured properly only in a treatment of the full ternary mixture. Moreover, we speculate that the topology is likely to hold for an analogous 3D system (hard-sphere colloids); there is nothing particular to two dimensions, an assertion supported by our mean-field treatment-see Supplemental Material [17].

We thank N. Wilding, D. Ashton, and A. Maciołek for stimulating discussions. J. R. E. and M. D. acknowledge financial support from a Nederlandse Organisatie voor Wetenschappelijk Onderzoek (NWO) VICI grant. N. T. and M. D. acknowledge financial support from an NWOECHO grant. J.R.E., N.T., and M.D acknowledge a NWO-EW grant for computing time in the Dutch supercomputer Cartesius. R. E. acknowledges financial support from the Leverhulme Trust.

*m.dijkstra1@uu.nl

[1] G. Flöter and S. Dietrich, Z. Phys. B 97, 213 (1995).

[2] M. E. Fisher and P. G. de Gennes, C.R. Acad. Sci. Paris B 287, 207 (1978).

[3] H. B. G. Casimir, Kon. Ned. Akad. Wetensch. Proc. 51, 793 (1948).

[4] M. Krech, The Casimir Effect in Critical Systems (World Scientific, Singapore, 1994).

[5] R. Evans and J. Stecki, Phys. Rev. B 49, 8842 (1994).

[6] A. Hanke, F. Schlesener, E. Eisenriegler, and S. Dietrich, Phys. Rev. Lett. 81, 1885 (1998).

[7] M. Krech, J. Phys. Condens. Matter 11, R391 (1999).

[8] O. Vasilyev, A. Gambassi, A. Maciołek, and S. Dietrich, Phys. Rev. E 79, 041142 (2009).

[9] C. Hertlein, L. Helden, A. Gambassi, S. Dietrich, and C. Bechinger, Nature (London) 451, 172 (2008).

[10] A. Gambassi, A. Maciołek, C. Hertlein, U. Nellen, L. Helden, C. Bechinger, and S. Dietrich, Phys. Rev. E 80, 061143 (2009).

[11] D. Beysens and D. Estève, Phys. Rev. Lett. 54, 2123 (1985).

[12] V. D. Nguyen, S. Faber, Z. Hu, G. H. Wegdam, and P. Schall, Nat. Commun. 4, 1584 (2013).

[13] T. F. Mohry, A. Maciołek, and S. Dietrich, J. Chem. Phys. 136, 224902 (2012).
[14] M. T. Dang, A. V. Verde, V. D. Nguyen, P. G. Bolhuis, and P. Schall, J. Chem. Phys. 139, 094903 (2013).

[15] T. F. Mohry, S. Kondrat, A. Maciolek, and S. Dietrich, Soft Matter, 10, 5510 (2014).

[16] E. Rabani, D. R. Reichman, P. L. Geissler, and L. E. Brus, Nature (London) 426, 271 (2003).

[17] See Supplemental Material at http://link.aps.org/ supplemental/10.1103/PhysRevLett.114.038301, which includes Refs. [18-23], for (a) results from a simple mean-field theory of the $A B C$ model, (b) details of our simulation methods, (c) investigations on lattice effects present in our model, (d) some results on structure of the ternary mixture, and (e) some movies of our simulations.

[18] F. A. Escobedo, J. Chem. Phys. 127, 174104 (2007).

[19] A. Santos, M. López de Haro, and S. Bravo Yuste, J. Chem. Phys. 103, 4622 (1995).

[20] D. A. Young and B. J. Alder, J. Chem. Phys. 70, 473 (1979).

[21] J. Hansen and I. McDonald, Theory of Simple Liquids, 3rd ed. (Academic, New York, 2006).

[22] M. Zubaszewska, A. Maciołek, and A. Drzewiński, Phys. Rev. E 88, 052129 (2013).

[23] T. W. Burkhardt and E. Eisenriegler, Phys. Rev. Lett. 74, 3189 (1995).

[24] E. P. Bernard and W. Krauth, Phys. Rev. Lett. 107, 155704 (2011).

[25] R. Evans, J. Phys. Condens. Matter 2, 8989 (1990).

[26] D. J. Ashton and N.B. Wilding, Mol. Phys. 109, 999 (2011).

[27] J. R. Errington, Phys. Rev. E 67, 012102 (2003).

[28] R. Evans, R. J. F. Leote de Carvalho, J. R. Henderson, and D. C. Hoyle, J. Chem. Phys. 100, 591 (1994).

[29] M. G. Noro and D. Frenkel, J. Chem. Phys. 113, 2941 (2000).

[30] G. A. Vliegenthart and H. N. W. Lekkerkerker, J. Chem. Phys. 112, 5364 (2000).

[31] A. Drzewiński, A. Maciołek, and R. Evans, Phys. Rev. Lett. 85, 3079 (2000).

[32] R. Okamoto and A. Onuki, J. Chem. Phys. 136, 114704 (2012).

[33] S. L. Veatch, P. Cicuta, P. Sengupta, A. Honerkamp-Smith, D. Holowka, and B. Baird, ACS Chem. Biol. 3, 287 (2008).

[34] B. B. Machta, S. L. Veatch, and J. P. Sethna, Phys. Rev. Lett. 109, 138101 (2012). 